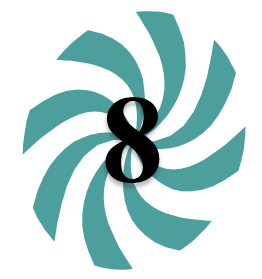

Tecnociencia, Vol. 22, $\mathrm{N}^{\circ} 2: 151-174$

julio-diciembre 2020

\title{
SITUACIÓN ACTUAL DE LAS ÁREAS PROTEGIDAS EN LA PROVINCIA DE COLÓN, PANAMÁ: MARCO DESCRIPTIVO PARA UNA MEJOR GESTIÓN Y MANEJO
}

${ }^{1}$ Octavio Ortiz, ${ }^{2,6}$ Yolanda Moreno de Niño, ${ }^{3}$ Ursula Vargas Cusatti, ${ }^{2,6}$ Vera De La Cruz Cabrera $\&^{\mathbf{4 , 5 , 6}}$ Alfredo Lanuza-Garay

${ }^{1}$ Ministerio de Ambiente de la República de Panamá. Departamento de Enlace de Costas y Mares. Correo electrónico: oortiz@miambiente.gob.pa.

${ }^{2}$ Universidad de Panamá, Centro Regional Universitario de Colón. Departamento de Ciencias Ambientales. Correo Electrónico: vera.delacruz@up.ac.pa, yolandamorenodenino@gmail.com.

${ }^{3}$ Universidad del Istmo. Catedra de Ecología y Medioambiente. Correo electrónico: ucusatti22@gmail.com.

${ }^{4}$ Universidad de Panamá, Centro Regional Universitario de Colón, Departamento de Zoología. Correo Electrónico: alfredo.lanusa@up.ac.pa.

${ }^{5}$ Museo de Invertebrados Graham Bell Fairchild, Universidad de Panamá.

${ }^{6}$ Sociedad Mesoamericana para la Biología y la Conservación

C) orcid.org/0000-0002-5142-4538

\section{RESUMEN}

Las áreas protegidas son por excelencia los lugares más valiosos para la conservación in situ de la naturaleza en un país. En Panamá, dichos espacios naturales son de interés institucional y de gestión por el beneficio ambiental que ofrecen, a pesar de la intensa presión humana de la cual son objeto. Al respecto, el Sistema Nacional de Áreas Protegidas (SINAP) del departamento de Manejo de Áreas Protegidas del Ministerio de Ambiente (MiAmbiente) de Panamá, través del Programa de Monitoreo de la Efectividad del Manejo de las Áreas Protegidas (PMEMAP) verifica todas a nivel nacional y su estado actual. El país cuenta con ciento cinco (105) áreas protegidas legalmente establecidas, de las cuales la provincia de Colón cuenta con cuatro: Parque Nacional Portobelo, Bosque Protector y Paisaje Protegido San Lorenzo, Área Recreativa Lago Gatún y Paisaje Protegido de Isla Galeta. Sin embargo, estas no 
cuentan desde 2012 con un análisis profundo que describa su particularidad en torno a aspectos sociales, ambientales, culturales y económicos, información que podría ser útil en la búsqueda de las posibles líneas de acción a corto, mediano y largo plazo. En esta investigación de carácter descriptivo sobre áreas protegidas de Colón y su situación actual, basándose en cinco ejes: social, administrativos, económicofinancieros, político-legal, recursos naturales y culturales, además de investigaciones científicas y amenazas registradas, se realizó trabajo de campo y se revisó información del departamento de Manejo Áreas Protegidas del Ministerio de Ambiente. Los resultados muestran realidades diversas en cada una de las áreas protegidas tratadas, se recomienda establecer nuevos planes de acción y alianzas estratégicas apoyadas en educación ambiental, producción y fortalecimiento del conocimiento científico e incremento de personal idóneo. Por otro lado, las amenazas registradas se muestra un incremento significativo, lo que sugiere una incidencia negativa en la integridad de dichas áreas.

\title{
PALABRAS CLAVES
}

Mi AMBIENTE, SINAP, Investigación Científica, Panamá, Gestión Ambiental.

\section{CURRENT STATUS OF PROTECTED AREAS IN COLON PROVINCE, PANAMA: A DESCRIPTIVE FRAMEWORK FOR BETTER MANAGEMENT}

\begin{abstract}
Protected lands are the most valuable places for in situ conservation of nature. In Panama, these natural spaces are of institutional and management interest due to the environmental benefit they offer, despite intense human pressure to which they are subject. In this sense, National System of Protected Areas (SINAP) of Department of Management of Protected Areas of the Ministry of Environment (MiAmbiente) of Panama, through the Program for Monitoring the Effectiveness of Management of Protected Areas (PMEMAP) verifies all these lands and its current status. Panama has 105 legally established protected areas, of which Colon province have four of them, Portobelo National Park, San Lorenzo Protector Forest and Protected Landscape, Gatun Lake Recreational Area and Galeta Island Protected Landscape. However, since 2012, they have not had an in-depth analysis that describes their particularity around social, environmental, cultural and economic aspects, information that could be useful in the search for possible short, medium and long term lines of action. In this descriptive research on Colon province protected areas and its current situation, based on five axes: social, administrative, economic-financial, political-legal, natural and cultural resources, as well as scientific research and protected land recorded threats,
\end{abstract}


field work was carried out and information from the Department of Protected Area Management of the Ministry of Environment was reviewed. The results show diverse realities in each of the protected areas treated, it is recommended to establish new action plans and strategic alliances supported by environmental education, production and strengthening of scientific knowledge and increase of qualified personnel. On the other hand, recorded threats show a significant increase, which suggests a negative impact on their integrity.

\section{KEY WORDS}

Mi AMBIENTE, SINAP, Scientific Research, Panamá, Environmental management

\section{INTRODUCCIÓN}

América Latina representa $10 \%$ de la superficie continental y marítima del globo terrestre, en la que alberga $65 \%$ de la diversidad biológica y cultural de la Tierra (Hockings et al., 2006). Los esfuerzos que actualmente realizan todos los países de la región latinoamericana han logrado consolidar la creación de alrededor de 4000 áreas protegidas, como una de las estrategias más eficientes para contribuir a la conservación de la biodiversidad y aportar a los objetivos del desarrollo sostenible de los pueblos de la región (Castaño-Uribe, 2007) Las áreas protegidas, tanto en América Latina como en todo el planeta, son los lugares más valiosos para la conservación in situ de la naturaleza (Ergueta et al, 1992). La preservación de estos tesoros que resguardan la diversidad de la vida nunca fue más evidente que hoy, porque nunca antes el ser humano y sus actividades económicas fueron una amenaza tan fuerte sobre la comunidad de la vida que a principios del siglo XXI (Elbers, 2011).

Este conjunto de áreas protegidas consolida una superficie mayor a cuatro millones de $\mathrm{Km}^{2}$, es decir, más de $18 \%$ del territorio terrestre de la región, y aproximadamente $20 \%$ de la extensión de las áreas protegidas del mundo. Estas cifras revelan que América Latina es una de las zonas geográficas más dinámicas y comprometidas con la designación y manejo de áreas protegidas en el mundo (Castaño-Uribe, 2007). 
Las evaluaciones realizadas señalan que en la última década las amenazas existentes a la integridad de las áreas protegidas se han intensificado, especialmente en aspectos concernientes al avance de actividades agrícolas de gran escala, colonización ilegal de tierras, la extracción ilegal de madera, la difusión de especies invasoras de flora y fauna, la incursión de actividades mineras, petroleras y forestales no sostenibles, la cacería y pesca ilegal, entre otras (Jones et al, 2018). En muchos casos, estas amenazas se ven incrementadas por la instalación de grandes iniciativas de infraestructura vial y energética que adolecen de consideraciones ambientales adecuadas, así como por el aumento de la demanda de recursos naturales para el comercio internacional, lo cual las hace más vulnerables al cambio global (Ergueta et al., 1992; Hockings et al., 2006; Arnold Torrez \& Barroso Pauletti, 2008; Elbers, 2011).

Panamá cuenta con 105 áreas protegidas legalmente establecidas, representando la mayor parte de los ecosistemas naturales del país con una superficie aproximada a los 2922,648 ha, es decir un 35.8\% del territorio nacional, donde la mayor parte esta categorizada como Parque Nacional y Reservas Forestales. (ANAM, 2011), sin embargo su monitoreo surge a partir de una iniciativa Centroamericana denominada "Estrategia de Monitoreo de las Áreas Protegidas", apoyada por el Programa Ambiental Regional para Centroamérica (PROARCA) en coordinación con el Consejo Centroamericano de Bosques y Áreas Protegidas (CCAB-AP) de la Comisión Centroamericana de Ambiente y Desarrollo (CCAD) (ANAM, 2008), programa que fue posteriormente adoptado por la entonces Autoridad Nacional del Ambiente (ANAM, hoy Ministerio de Ambiente de Panamá) como la herramienta de importancia para la gestión por parte del Sistema Nacional de Áreas Protegidas (ANAM, 2008).

Aun cuando se cuenta con documentos analíticos sobre las condiciones de las áreas protegidas de todo el país, llama la atención que las áreas protegidas de la provincia de Colón después del 2012 no muestran un análisis profundo que describa su particularidad en torno a los aspectos sociales, ambientales, culturales y económicos, información que podría ser útil en la búsqueda de las posibles líneas de acción a corto, mediano 
y largo plazo, con organizaciones gubernamentales y $\mathrm{ONG}^{\prime}$ s para su mejor gestión.

Ante este marco, esta investigación tiene como propósito diagnosticar la situación actual de las diferentes áreas protegidas con que cuenta la provincia de Colón y establecer posibles líneas de acción para el fortalecimiento de su gestión, determinando la superficie total de territorio bajo protección y su estado de conservación, revisando los niveles de gestión de cada área protegida tratada, el estado de la investigación científica desarrollada en ellas, así como las diferentes amenazas que afrontan y de esta manera sugerir líneas de acción para el fortalecimiento de su gestión y manejo.

\section{MATERIALES Y METODOS}

El estudio presenta una estructura longitudinal, permitiendo el seguimiento de situaciones y factores estadísticamente medibles o comparables a través del tiempo, ofreciendo indicadores más precisos de los cambios que presentan los factores estudiados, además, de permitir que sus consecuencias puedan aplicarse en otros campos de las ciencias (Delgado Rodríguez \& Lorca Díaz, 2004, Sampieri et al., 2010). El mismo se desarrolló con información correspondiente desde el 2009 hasta el 2018.

Se tomaron en cuenta las siguientes áreas protegidas de la provincia de Colón: Parque Nacional Portobelo, Parque Nacional San Lorenzo, Área Recreativa Lago Gatún y Paisaje Protegido de Isla Galeta, sin embargo, no se tomaron en cuenta las áreas protegidas de Donoso (Categoría de Área de Uso Múltiple) y Santa Isabel (Categoría de Uso Múltiple y Reserva Hidrológica), debido a que no cuentan con infraestructura adecuada ni planes de manejo.

Para un mejor entendimiento del manejo y gestión de las áreas protegidas tratadas, se procedió a una revisión exhaustiva de los resultados de evaluación de PMEMAP de 2009 a 2012, debido a que posterior a esta fecha no se han realizado otras evaluaciones a las áreas 
protegidas en cuestión, focalizadas principalmente a áreas protegidas de interés prioritario (programas de captura de Carbono, canje de deudas por naturaleza entre otros), asi como de los planes de manejo de las áreas protegidas evaluadas (AECI et al., 1992; ANAM et al., 2002; ANAM, 2002; ANAM et al., 2013), planes operativos anuales (POA), estadísticas de Investigaciones realizadas en las áreas seleccionas desde los años 2014 a 2018; decretos ejecutivos, decretos ley que crean o regulan estas áreas (AL, 1976; 1992; INRENARE, 1994, MEF, 2013; ANAM, 2013,2017), de igual forma se realizaron visitas a las áreas protegidas tratadas para documentar la situación in situ de dichas áreas y contrastarla con la información recabada.

La evaluación actual de las áreas protegidas de la Provincia de Colón se realizó basada en cinco ejes principales:

\section{Nivel de gestión Social}

En este ámbito se dispone de indicadores para medir las relaciones y actividades que se realizan en el marco de la gestión del área protegida con las comunidades, instituciones de gobierno, organizaciones de la sociedad civil, empresas y visitantes.

\section{Nivel de gestión administrativa}

Incluye indicadores que permiten medir el manejo institucional, tales como: infraestructuras, equipo, personal y la planificación a corto y largo plazo.

\section{Nivel de Gestión de recursos naturales y culturales}

Los indicadores que forman parte de este ámbito están relacionados al estado de conservación del área protegida, la relación entre el aprovechamiento compatible y/o no compatible con los objetos de creación del área protegida, programas de vigilancia, investigación y monitoreo ambiental. En ese sentido, se compiló información de las investigaciones registradas y realizadas en las áreas protegidas a tratar en este trabajo, de 2016 hasta 2019. Este elemento permite hacer un análisis crítico de la gestión del AP desde la perspectiva de gestión de los recursos naturales, así como las entidades que realizan dichas investigaciones. 


\section{Nivel de gestión político-legal}

Este ámbito cuenta con dos indicadores sobre el marco legal e institucional de las áreas protegidas.

\section{Nivel de gestión económico-financiero}

Este ámbito cuenta con indicadores relacionados a la evaluación del proceso de planificación y disponibilidad financiera del área protegida y su capacidad de recibir beneficios por los bienes y servicios que genera a la sociedad.

Una vez tomada la información de cada una de las áreas protegidas en estos cuatro ámbitos, se procedió a evaluar numéricamente la gestión de manejo a partir de una escala de manejo, que no es más que la calificación que obtiene el área protegida en un ámbito o en su gestión general, en una escala de cero a 100 puntos. Esta calificación es calculada por la base de datos en donde la evaluación de los indicadores y de los ámbitos de la gestión tiene el mismo peso. Las calificaciones de cada ámbito se realizan de acuerdo al máximo posible del puntaje de cada indicador. Por lo tanto, el porcentaje de gestión dado por el nivel de calificación para el área protegida en su conjunto está definido por el máximo total de puntos posibles (i.e. la sumatoria de todos los indicadores con calificación de 5 dividido por la evaluación obtenida de todos los indicadores del ámbito correspondiente), donde la sumatoria de los indicadores indicaran los niveles de gestión: 0-200 (No aceptable), 201-400 (Poco aceptable), 401-600 (Regular), 601-800 (Aceptable) y más de 800 (Satisfactorio).

En el caso de aquellos indicadores que no apliquen a las condiciones del área protegida (ejemplo: indicadores relacionados con el ecoturismo) simplemente se excluyen de las estimaciones sin afectar la calificación de los demás indicadores.

\section{RESULTADOS}

La provincia de Colón cuenta con un total de 276549.60 hectáreas bajo protección, de las cuales 47809.68 ha corresponden a las cuatro áreas gestionadas bajo administración del Ministerio de Ambiente 
seleccionadas para este estudio.

Gestión de las Áreas Protegidas de la Provincia de Colón Las áreas protegidas de la provincia de Colón de acuerdo al PMMAP de 2012, obtuvieron un total de 579.04 puntos, lo que las ubica en un nivel de valorización regular en cuanto a su manejo. El Área Protegida con mayor valorización obtenida fue el Bosque Protector y Paisaje Protegido San Lorenzo mientras que la más baja la obtuvo el Parque Nacional Portobelo (Cuadro 1)

Cuadro 1. Valorización de las Áreas protegidas de la Provincia de Colón, de acuerdo a PMEMAP 2012.

\begin{tabular}{lcccccc}
\hline Área Protegida & Social & Administrativo & $\begin{array}{c}\text { Recursos } \\
\text { Naturales }\end{array}$ & $\begin{array}{c}\text { Político- } \\
\text { Social }\end{array}$ & Económico & Total \\
\hline PN Portobelo & 376 & 642 & 586 & 525 & 55 & $\mathbf{4 4 0}$ \\
BPPP San Lorenzo & 727 & 674 & 628 & 881 & 598 & $\mathbf{7 0 7}$ \\
PP Isla Galeta & 788 & 543 & 539 & 631 & 47 & $\mathbf{5 1 2}$ \\
AR Lago Gatún & 563 & 604 & 549 & 881 & 744 & $\mathbf{6 0 7}$ \\
Total & $\mathbf{6 1 3 . 5}$ & $\mathbf{6 1 5 . 8}$ & $\mathbf{5 7 5 . 5}$ & $\mathbf{7 2 9 . 4}$ & $\mathbf{3 6 1}$ & \\
\hline
\end{tabular}

Fuente: Elaborada con datos proporcionados por los Resultados del PMEMAP 2012

Por criterio de evaluación (social, administrativo, recursos naturales, político social y económico), las áreas protegidas de Colón presentan realidades diversas. En el ámbito social Portobelo se valoriza en un nivel de gestión poco aceptable, el Área Recreativa Lago Gatún en un nivel regular, mientras que el Bosque Protector y Paisaje Protegido San Lorenzo y el Paisaje Protegido de Isla Galeta indican un nivel de gestión social en el marco de lo aceptable.

En el ámbito administrativo, las áreas protegidas (AP), mostraron niveles de gestión que van desde regular a satisfactorio, obteniendo el mayor puntaje el Parque Nacional San Lorenzo, mientras el Paisaje Protegido Isla Galeta obtuvo el puntaje más bajo. En cuanto al manejo de los recursos naturales, las áreas protegidas de la provincia de Colón primordialmente se encuentran valorizados dentro de una gestión 
regular, sólo el Bosque Protector y Paisaje Protegido San Lorenzo, obtuvo una valorización que la ubica dentro del rango aceptable.

En el aspecto político social, las AP de la provincia de Colón se ubican en la valorización de gestión aceptable, siendo el BPPP San Lorenzo y el Área Recreativa Lago Gatún las que mayor puntaje obtuvieron, mientras que el Parque Nacional Portobelo obtuvo los valores más bajos.

La gestión de las áreas protegidas de la provincia de Colón a partir del PMEMAP del 2009 hasta 2012, muestran mejorías significativas respecto a los valores anuales de dicho instrumento, mostrando valores poco aceptables en 2010 a niveles aceptables de manejo para el 2012 (Figura 1, Cuadro 2)

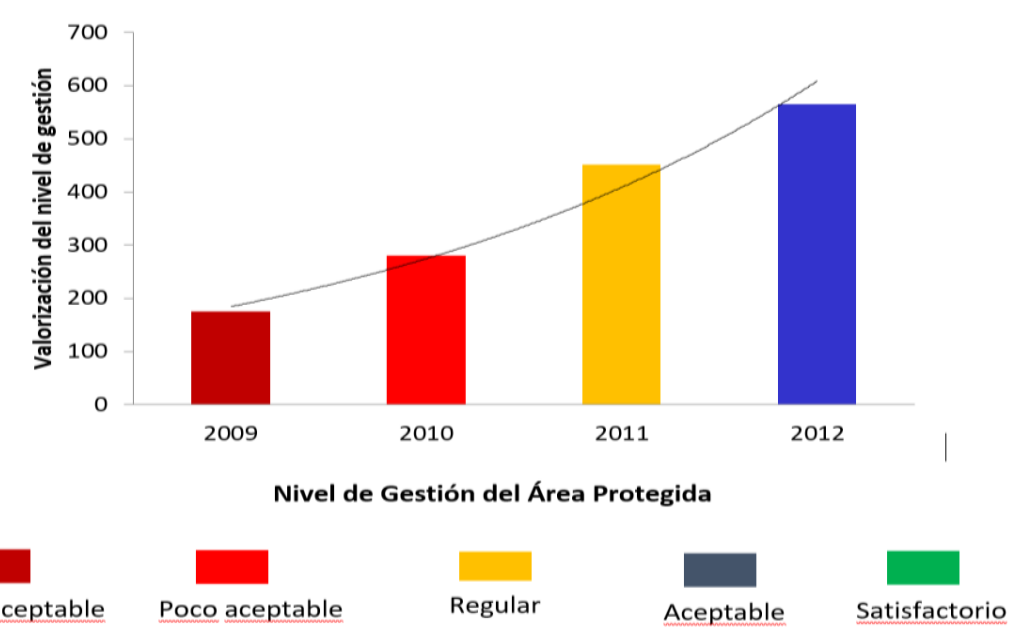

Fig. 1 Nivel de gestión de las áreas protegidas de la Provincia de Colón, de acuerdo a la valorización del PMEMAP desde 2009 a 2012 
Cuadro 2. Valorización de las Áreas protegidas de la Provincia de Colón, de acuerdo a PMEMAP 2009-2012

\begin{tabular}{lcccc}
\hline Área Protegida & $\mathbf{2 0 0 9}$ & $\mathbf{2 0 1 0}$ & $\mathbf{2 0 1 1}$ & $\mathbf{2 0 1 2}$ \\
\hline PN Portobelo & 112 & 186 & 392 & 440 \\
BPPP San Lorenzo & 193 & 273 & 524 & 707 \\
PP Isla Galeta & 188 & 295 & 452 & 512 \\
AR Lago Gatún & 212 & 370 & 441 & 607 \\
Total & $\mathbf{1 7 6}$ & $\mathbf{2 8 1}$ & $\mathbf{4 5 2}$ & $\mathbf{5 6 6}$ \\
\hline
\end{tabular}

Fuente: Resultados de los PMEMAP 2009, 2010, 2011 y 2012

La investigación científica en las áreas protegidas de la provincia de Colón

Las áreas protegidas de la provincia de Colón presentan un aspecto negativo, respecto al desarrollo de este aspecto en otras áreas protegidas a nivel nacional, desarrollándose solo un $12.6 \%$ de toda la investigación en las AP a nivel nacional, lo que representa 240 investigaciones registradas de casi 2000 en todo el país (Figura 2).

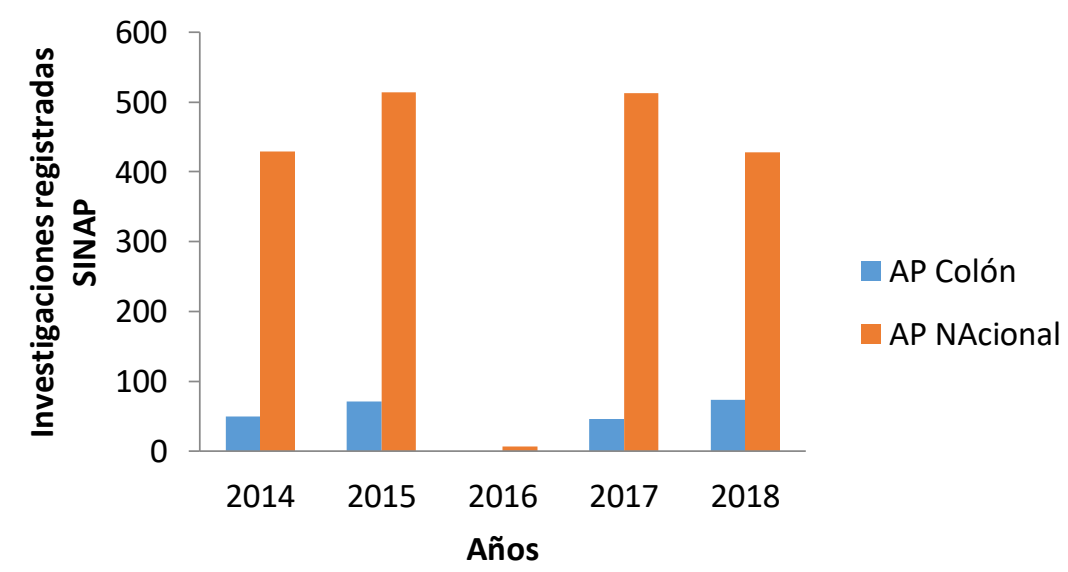

Fig. 2 Comparación del número de investigaciones realizadas y registradas en las Áreas Protegidas de la Provincia de Colón respecto al número de investigaciones registradas a nivel nacional del 2014 al 2018 
En cuanto a la investigación dentro de las áreas protegidas en la provincia de Colón, se observa que la mayor parte se desarrolla en el Bosque Protector y Paisaje Protegido San Lorenzo, mientras que en la que menos se desarrollan programas de investigación es en el Área Recreativa Lago Gatún (Figura 3) Entre los taxones más abordados en las investigaciones realizadas en las AP, el $59 \%$ de ellas son taxones zoológicos, siendo los más estudiados los invertebrados, mientras que las plantas ocupan el segundo lugar con un $31 \%$. Por otro lado, taxones como fungi (hongos) y bacterias son poco estudiados en las AP (Figura 4).

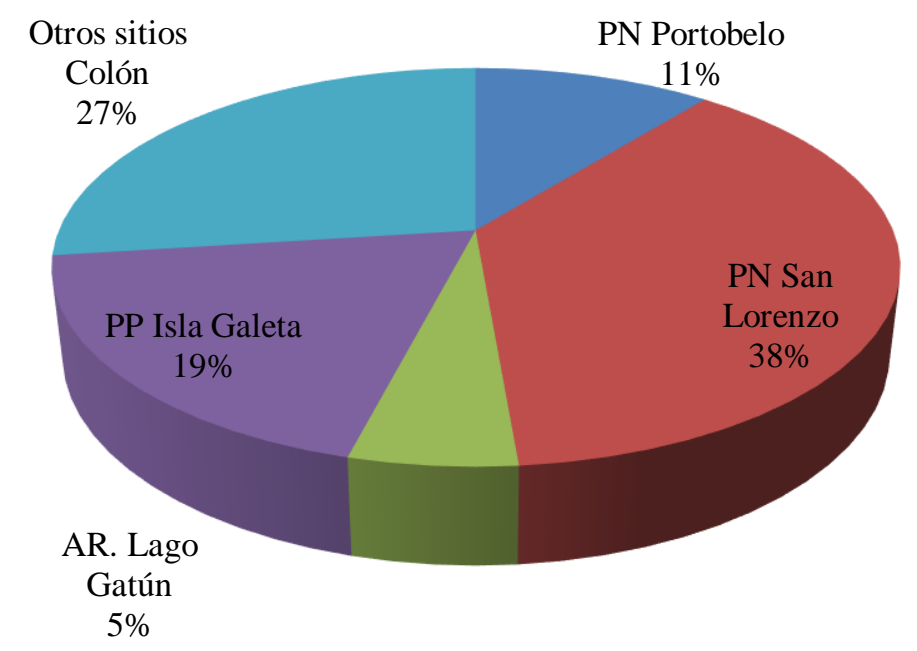

Fig. 3 Porcentaje de investigaciones registradas en las diferentes Áreas Protegidas de la Provincia de Colón entre los años 2014 y 2018. 


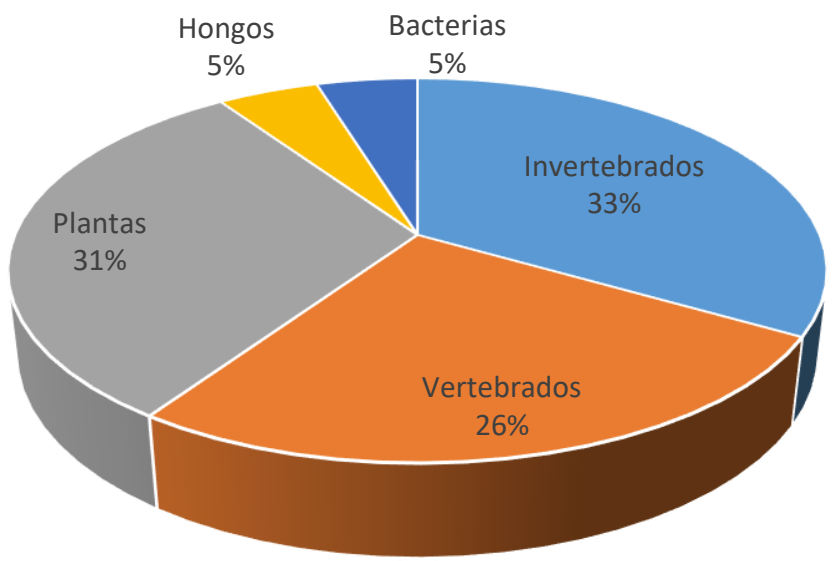

Fig. 4 Porcentaje de taxones tratados en las investigaciones realizadas en las diferentes Áreas Protegidas de la Provincia de Colón entre los años 2014 y 2018

Por su parte, el 56\% las áreas temáticas en las que se enmarcan las investigaciones realizadas en las AP de la provincia de Colón son de índole Ambiental y Ecológico, mientras que sólo un 15\% están enfocadas al estudio de la biodiversidad (Figura 5).

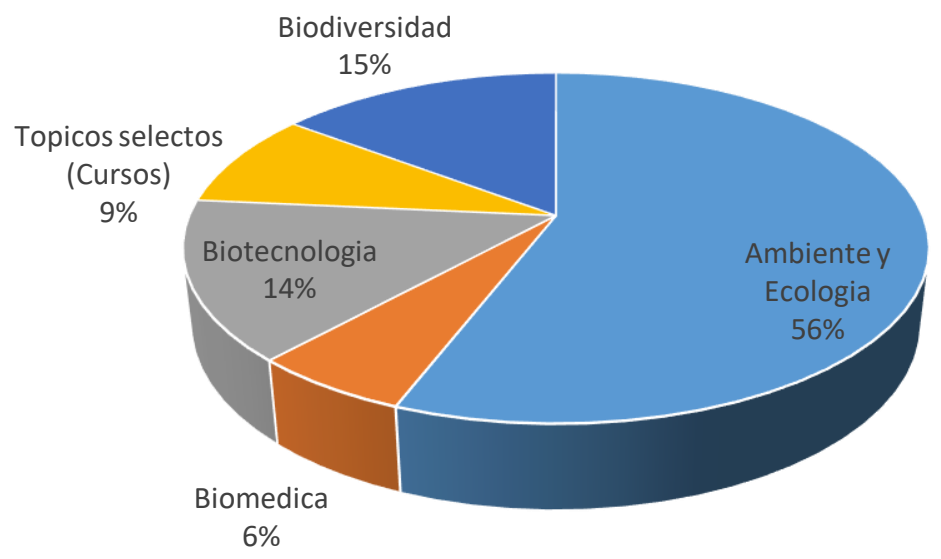

Fig. 5 Áreas Temáticas tratadas en las investigaciones realizadas en las diferentes Áreas Protegidas de la Provincia de Colón entre los años 2014 y 2018 
Para el desarrollo de estas investigaciones, los investigadores nacionales o internacionales, están respaldados por diferentes instituciones, en este caso, $76 \%$ de las investigaciones realizadas en las áreas protegidas de la provincia de Colón, están respaldadas por el Instituto Smithsonian de Investigaciones Tropicales (STRI), mientras que sólo un 9\% es respaldada por la Universidad de Panamá, mientras que 15\% está respaldada por otras instituciones como la Sociedad Panameña de Mastozoología (SOMASPA), El Instituto Conmemorativo Gorgas de Estudios de la Salud(ICGES), El Centro de Investigaciones Biológicas el Copé, El Instituto de Investigaciones Científicas y Servicios de Alta Tecnología (INDICASAT), la Universidad Marítima de Panamá (UMIP), la Sociedad de Biología de Panamá, así como investigaciones de carácter independiente (Figura 6).

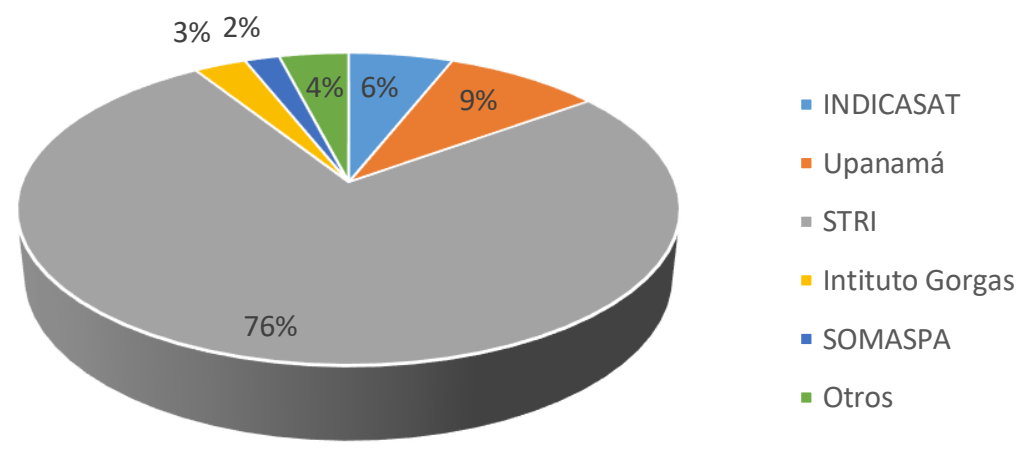

Fig. 6 Porcentaje de investigaciones realizadas por institución en las áreas protegidas de la Provincia de Colón

Evaluación de los Instrumentos de Gestión de las Áreas Protegidas en la Provincia de Colón

De acuerdo a la información recabada durante esta investigación, todas las áreas protegidas de la provincia de Colón, a la fecha, cuentan con sus planes operativos anuales (POA) en activo, sin embargo, no son 
aplicadas en su totalidad, ya que dependen de la administración de fondos por parte de la sede central del Ministerio de Ambiente, en cuanto a los PMEMAP, no se han realizado más evaluaciones de gestión en las áreas protegidas de la Provincia de Colón desde 2012, sin embargo, se documenta la realización de estas evaluaciones de gestión en otras áreas protegidas del país, como lo es el Parque Nacional Darién, el Parque Nacional Alto Chagres, el Parque Nacional Coiba y el Parque Nacional Altos de Campana, debido a su importancia y valor dentro de la gestión institucional de los AP a nivel nacional e internacional.

\section{Principales amenazas de las Áreas Protegidas de la Provincia de Colón}

Dentro de las principales amenazas para la conservación, protección y manejo de las áreas protegidas en Colón, (cuadro 3) que se pudieron evidenciar al momento de revisar los resultados de los PMEMAP, se pueden mencionar la ganadería extensiva, la extracción selectiva de recursos, la invasión de tierras dentro de las áreas protegidas, además de problemas relacionados al manejo compartido de las áreas protegidas.

Cuadro 3. Principales amenazas de las Áreas Protegidas de la Provincia de Colón

\begin{tabular}{lcccc}
\hline Amenazas/Área Protegida & PN Portobelo & PN San Lorenzo & PP Isla Galeta & AR Lago Gatún \\
\hline Planes de manejo desactualizados & & & $\mathrm{X}$ & \\
Extracción Selectiva de Recursos & $\mathrm{X}$ & $\mathrm{X}$ & $\mathrm{X}$ & $\mathrm{X}$ \\
Ganadería Extensiva & $\mathrm{X}$ & $\mathrm{X}$ & $\mathrm{X}$ & $\mathrm{X}$ \\
Invasión de Tierra & $\mathrm{X}$ & $\mathrm{X}$ & $\mathrm{X}$ & \\
Tensiones en el co-manejo de AP & $\mathrm{X}$ & $\mathrm{X}$ & $\mathrm{X}$ & $\mathrm{X}$ \\
Falta de personal idóneo & $\mathrm{X}$ & $\mathrm{X}$ & $\mathrm{X}$ & $\mathrm{X}$ \\
Falta de equipos/gestión de AP & $\mathrm{X}$ & & $\mathrm{X}$ & $\mathrm{X}$ \\
Tenencia de Tierras & & $\mathrm{X}$ & $\mathrm{X}$ \\
Ausencia de programas de vigilancia y & $\mathrm{X}$ & &
\end{tabular}

Fuente: Elaboración propia de acuerdo a información recabada en las 4 áreas protegidas

En las áreas protegidas de Portobelo se evidencian problemas con la ganadería extensiva, el aumento de potreros cada vez más cerca, o incluso dentro de los límites del PN Portobelo motivados por la insostenibilidad de los sistemas productivos existentes, lo que 
contribuye a la degradación del recurso, motivando además la migración agraria. Además, se puede mencionar los problemas en cuanto al manejo compartido o co-manejo entre los actores (Instituto Nacional de Cultura, Ministerio de Salud, Municipio de Portobelo, Mi Ambiente, Autoridad Marítima de Panamá, Patronato Portobelo-San Lorenzo), donde se evidencia la falta de sinergias adecuadas para lograr el manejo eficiente del área protegida. Otro de los problemas evidenciados es la falta de personal y de equipo motorizado, lo que dificulta la aplicación del plan de manejo, además se evidencia la falta de infraestructuras, ya que sólo se cuenta con la caseta de vigilancia, la casa de guardaparques y un depósito.

El Área Recreativa Lago Gatún presentó evidencias de problemas en cuanto a la tenencia de tierras, especialmente con las vecindades del ARLG, debido a la construcción de la Autopista Panamá-Colón, a raíz de esta situación se documentó el fenómeno de invasión de tierras en el área de Villa del Caribe, cambiando su estatus de áreas de protección absoluta a zona cultural activa, mediante resolución aprobada en el plan de manejo de 2013, sin embargo, en la praxis esto no está resuelto, además de ser un problema urbanístico por los efectos ambientales que causan en el área protegida como lo es el efecto de borde.

El Paisaje Protegido de Isla Galeta presenta como principal amenaza la usencia de un plan de manejo actualizado, el cual data del año 2002, por otra parte, la construcción y puesta en funcionamiento del proyecto residencial Altos de los Lagos, se documenta el mal aprovechamiento de los recursos presentes, igualmente la extracción de recursos naturales marino-costeros (pesquería ilegal y extracción de cangrejos). Es importante mencionar que al igual que el PN Portobelo, el PP de Isla Galeta, presencia deficiencias en cuanto a personal, lo que dificulta aplicar lo señalado en los planes de manejo, programa de control y vigilancia y educación ambiental. En el caso de Isla Galeta, de acuerdo al plan de manejo, los actores que conforman el manejo del área protegida, serian el municipio de Colón, El Instituto Smithsonian de Investigaciones Tropicales, La Universidad de Panamá, La Universidad Tecnológica de Panamá, así como el Ministerio de Ambiente, de los 
cuales sólo dos se han mantenido con actores de beligerancia en el proceso, MiAMBIENTE y STRI.

Por otra parte, el Bosque Protector y Paisaje Protegido San Lorenzo presenta amenazas de diversa índole, desde la invasión de tierras hasta el mal aprovechamiento de los recursos. La invasión de territorios se evidencia en la región de Achiote Sur, cercano a la comunidad de Achiote, el área de Loma Flores, perteneciente a la comunidad de Escobal, siendo una de las problemáticas más importantes del área sumada a la tala indiscriminada (aprox. 85 ha entre Achiote sur y Loma Flores y aprox. 60 ha en el área de la 35), de igual forma la extracción de cangrejos en el área de la carretera hacia Sherman.

En el caso de San Lorenzo, que tiene un fortalecido manejo compartido, se anexa otro actor importante que son organizaciones de base comunitaria (OBC) para efectos de que la comunidad obtenga beneficios ecosistémicos de las áreas protegidas para diversos fines, sin embargo, aún se observan prácticas como la tala, la cacería ilegal dentro de estas áreas, que atentan contra los recursos y la integridad misma de las áreas protegidas evaluadas.

Al revisar los resultados de los PMEMAP realizados por MiAMBIENTE, se detectó la presencia de otras amenazas identificables dentro de algunas de las áreas protegidas de la provincia de Colón, el Parque Nacional San Lorenzo cuenta con un programa de control y vigilancia (patrullajes), sin embargo, el mismo debe ser actualizado. El caso de Isla Galeta es complejo, ya que no cuenta con las señalizaciones límites y zonas de amortiguamiento, lo que aumenta la potencialidad de amenazas de índole ambiental, especialmente la introducción de agentes externos (cazadores, pesca ilegal, extracción de recursos, extracción hierro de antiguas instalaciones militares).

\section{DISCUSIÓN}

La necesidad de bienes materiales y servicios que tiene la humanidad solamente puede ser atendida con el aprovechamiento de los recursos naturales, a través del empleo de los servicios ecosistémicos que 
producen los diversos hábitats (Pavón-Zamora et al., 2008; DávalosSotelo, 2016). Este aprovechamiento ejerce un gran impacto sobre dichos recursos, renovables o no, lo que genera una gran afectación a los ecosistemas, pues, entre mayor es la población que debe ser satisfecha, mayor es la extracción.

La motivación para crear y mantener estas áreas es cumplir con los objetivos de preservar en su condición original, ejemplos típicos de los diversos ecosistemas para conocimiento, uso y disfrute de las generaciones futuras. Y entonces surge de inmediato la pregunta ¿qué áreas proteger y de qué tamaño deben ser esas áreas para que tengan posibilidades reales de subsistir y resistir las inevitables presiones a las que se verán sometidas? Es ahí donde entra en juego la investigación científica para abordar esta y otras preguntas de igual relevancia. Las áreas silvestres protegidas constituyen un recurso científico de importante valor debido a la gran cantidad de información científica que pueden generar. Es por eso que la investigación en sí misma, es otro objetivo principal de conservación y manejo, al proteger y conservar áreas naturales en Parques Nacionales y otras áreas silvestres protegidas del país, basado en las experiencias de Maass et al. (2010), en las áreas protegidas de México o las de Dávalos-Sotelo (2016) en cuanto a la creación de áreas protegidas basadas en el aspecto científico.

La investigación científica es un aspecto relevante de la gestión de las áreas protegidas, como lo establecen los distintos planes de manejo, y que son evaluadas en los planes de gestión de las AP, esta investigación científica debe estar sujeta a las necesidades que identifica el plan de manejo. En el caso de las áreas protegidas de Colón, el número de investigaciones realizadas en las áreas protegidas de la provincia de Colón es considerablemente bajo respecto al resto de áreas protegidas (12.6\% de las investigaciones registradas en AP a nivel nacional).

El tema desarrollo vs conservación sigue y seguirá vigente mientras existan necesidades humanas que atender. El esquema que ha probado ser el más adecuado para buscar un equilibrio entre ambas partes del dilema es el de crear y mantener reservas protegidas. ¿Cómo seleccionar 
estas áreas? Es indiscutible que la investigación científica desempeña un papel fundamental en el proceso de decisión, junto con otros factores. Como ejemplo, en el tema de áreas protegidas con manglares y humedales de América tropical, Lugo y Snedaker (1974), Lugo y Cintron (1975) y Lugo et al. (2014), han hecho relevantes contribuciones, así como los trabajos de Sousa et al. (2003, 2007) utilizados como referencia para estudios e investigaciones que han servido como soporte científico para la creación y/o gestión de áreas de humedales protegidos.

En consenso, las áreas protegidas no están exentas de importantes problemas que amenazan constantemente la estabilidad de estos ecosistemas. En las áreas protegidas de la provincia de Colón se detectan innumerables amenazas, entre las que se mencionan la deforestación y el crecimiento de la frontera agropecuaria, especialmente en áreas protegidas como San Lorenzo y Portobelo, son considerados prácticamente por todos los países como el principal problema que enfrentan las áreas protegidas, por lo que todo esfuerzo encaminado a establecer líneas de acción debe contar con un entorno político, socioeconómico transparente en donde la planificación es imprescindible, así como la educación ambiental. El uso del conocimiento científico y las nuevas tecnologías de manejo, personal idóneo y planes estratégicos (Medrano \& Hernández, 2017).

Para una adecuada gestión y manejo de las áreas protegidas de la provincia de Colón se debe priorizar el manejo por objetivo de conservación en cuanto al tema de la gobernanza, se sugiere adecuar la coadministración para que las áreas sean gestionadas por asociaciones público-privadas, ello es así, ya que los gobiernos no destinan los recursos suficientes para su manejo, esta asociación deberá estar regulada y ser sujeto de controles, evaluaciones y/o auditorias.

Por otra parte, el Estado deberá dejar bajo su administración aquellas áreas que representen interés estratégico en cuanto al manejo de recursos y bienestar público, como son las cuencas hidrográficas que abastecen los centros urbanos (caso AR Lago Gatún) y las áreas de la cuenca del Canal de Panamá (Bosque Protector y Paisaje Protegido San Lorenzo), 
que son hábitat de especies endémicas o de interés, así como lo son las áreas protegidas con componente marino costero (Paisaje Protegido Isla Galeta y Parque Nacional Portobelo).

Además, se debe incentivar mecanismos de fideicomisos por área protegida con aportes nacionales y de fuente externa, mientras que el turismo bajo controles adecuados de capacidad de carga y respetando la zonificación puede representar - una fuente de recursos importante.

\section{CONCLUSIÓN}

La situación actual de las áreas protegidas de la provincia de Colón es compleja, aunque la provincia cuenta con una mayor superficie terrestre y marina protegida, con la adición de las áreas de uso múltiple de Donoso y Santa Isabel, lo cierto es que el nivel de gestión administrativa, socioeconómica, político legal y de recursos, distan de los estándares establecidos por el programa de monitoreo de Áreas Protegidas del Ministerio de Ambiente, a su vez, los instrumentos de medición de dicha gestión, aunque son los adecuados, en algunos casos no toman en cuenta las falencias administrativas, que de una manera $u$ otra inciden en el manejo deficiente de dichas áreas, por otra parte, la investigación científica, que debe ser el bastión fundamental para el manejo y gestión adecuada de las áreas protegidas, muestra números muy bajos además de que en muchos de los casos no representan los interés destacados en el plan de manejo de cada área.

Las múltiples amenazas a las que se enfrentan estas áreas protegidas, son variadas e inclusive son reiterativas no solo en la provincia de Colón, sino a lo largo de la geografía panameña, trayendo consigo un deterioro gradual e importante de ellas a corto, mediano y largo plazo.

De acuerdo a la información recabada y analizada se puede sustentar que los actores vinculados al manejo y gestión de las áreas protegidas de la provincia de Colón, en general han desatendido sus funciones en la continuidad de la consolidación de las estrategias de conservación, preservación así como la debida gestión de los bienes naturales 
comunes que ofrecen cada uno de ellos, por ende la planificación, el uso del conocimiento científico generado en las áreas protegidas como pilar de gestión, mejoramiento de la gobernanza y comanejo, el incentivo financiero y el interés administrativo del estado en la gestión adecuada de estas áreas permitirán, no solamente un manejo y gestión eficiente, sino también participativa y equitativa de las áreas protegidas estudiadas en esta investigación.

\section{AGRADECIMIENTOS}

Los autores agradecen al Ministerio de Ambiente de Panamá, específicamente a Braulio Correa por proporcionarme los resultados de los PMEMAP 2009, 2010, 2011 y 2012; a Jorge García, Alexander Montero, Carmen Medina y Anthony Vega, quienes proporcionaron el cuadro de las investigaciones realizadas dentro las áreas protegidas de Colón; de forma muy especial, también agradecemos a los jefes de las Áreas Protegidas de la Provincia de Colón, Edwin Guevara, Raúl Mariota, Luis Torres, por sus valiosos aportes con respecto a la situación actual de las áreas.

\section{REFERENCIAS}

Agencia Española de Cooperación Internacional (AECI), Instituto Nacional para la conservación de la Naturaleza (ANCON), Instituto Nacional de Recursos Renovables de Panamá (INRENARE) y Ministerio Publico (MP). 1992. Parque Nacional Portobelo, Plan de Manejo: Fase 1.

Arnold Torrez, I. y P. Barroso Pauletti. 2008. Áreas Protegidas de Bolivia: Situación y Perspectivas de Gestión. Nativa-Avina. Tarija, Bolivia.

Asamblea Legislativa de la República de Panamá. 1999. Decreto Ejecutivo 43 donde se reglamentan los capítulos II y III de la ley 91 del 22 de diciembre de 1976 y se establece el ordenamiento territorial del Parque Nacional de Portobelo y el conjunto monumental histórico de Portobelo 
Asamblea Legislativa de la República de Panamá. 1976.. Ley 91 del 22 de diciembre por la cual se regulan los conjuntos monumentales históricos de Panamá Viejo, Portobelo y el Casco Antiguo de la Ciudad de Panamá.

Autoridad Nacional de Ambiente (ANAM) y Unión Mundial para la Naturaleza (UICN). (2006). Estado de la Gestión Compartida de las Áreas Protegidas de Panamá. Disponible en el sitio web https://portals.iucn.org/library/sites/library/files/documents/2006008.pdf

Autoridad Nacional de Ambiente de Panamá (ANAM), Fundación Natura, Sistema Nacional de Áreas Protegidas de Panamá (SINAP). 2012. Informe del Programa de Efectividad de Manejo de las Áreas Protegidas del SINAP. 89 pp.

Autoridad Nacional de Ambiente de la República de Panamá (ANAM). 2013. Resolución AG-0647 del 1 de octubre de 2013, por la cual se aprueba el plan de manejo del Área recreativa Lago Gatún.

Autoridad Nacional del Ambiente (ANAM), Constructora Odebrecht y Corporación de Desarrollo Ambiental S.A. (CODESA). 2013. Plan de Manejo Área Recreativa Lago Gatún. 122 pp.

Autoridad Nacional del Ambiente de Panamá (ANAM), Autoridad de la Región Interoceánica (ARI), Instituto Nacional de Cultura (INAC), Instituto Panameño de Turismo (IPAT) y Consultores Ecológicos Panameños (CEPSA). 2002. Plan de Manejo del Área Protegida San Lorenzo. $180 \mathrm{pp}$.

Autoridad Nacional del Ambiente de Panamá (ANAM) (2002). Plan de Manejo del Paisaje Protegido de Isla Galeta. 49 pp

Cabrera, M., M. Gallardo y J. Santamaría. 2008. Informe 2006-2007. Programa de Monitoreo de la Efectividad del Manejo de las Áreas Protegidas del SINAP. Autoridad Nacional del Ambiente, Dirección de 
Áreas Protegidas y Vida Silvestre. 64 pp.

Cabrera, M. 2012. Informe PMEMAP 2012. Programa de Efectividad de Manejo de las Áreas Protegidas del SINAP. Autoridad Nacional del Ambiente, Dirección de Áreas Protegidas y Vida Silvestre. 89 pp.

Castaño-Uribe, C. 2007. Diagnóstico y situación actual de las áreas protegidas en América Latina y el Caribe 2007. Informe Regional. Plural editores, Bariloche, Argentina.

Dávalos-Sotelo, R. 2016. El papel de la investigación científica en la creación de las áreas naturales protegidas. Madera y bosques, 22(1), 713.

Delgado Rodríguez, M. y J. Llorca Díaz. 2004. Estudios Longitudinales: Concepto y Particularidades. Revista Española de Salud Pública 78 (2):141-148.

Díaz, F. 2018. Áreas Protegidas: Condición de producción para el desarrollo sostenible. Diario la Estrella de Panamá. Disponible en el sitio web: http://laestrella.com.pa/panama/nacional/areas-protegidascondicion-produccion-para-desarrollo-sostenible/24076439

Elbers, J. 2011. Las áreas protegidas de América Latina: Situación actual y perspectivas para el futuro. Quito, Ecuador, UICN, 227 p.

Ergueta, P., W. Hanagarth y M. Moraes. 1992. Situación actual y perspectiva de la Investigación Biológica en las áreas protegidas de Bolivia. The George Wright Forum, 9 (3/4): 20-31.

Hernández Sampieri, R., C. Fernández Collado, L. Baptista Lucio. 2010. Metodología de la Investigación. 5ta Edición. Editorial McGraw-Hill. $613 \mathrm{pp}$.

Hockings, M., S. Stolton, F. Leverington, N. Dudley, J. Courraeu. 2006. Evaluating Effectiveness: A Framework for Assesing Management effectiveness of Protected Areas. $2^{\text {nd }}$ edition. UICN Best Practice 
Protected Areas Series $N^{\circ}$ 14. Switzerland and Cambridge UK. 105 pp.

Instituto Nacional de Recursos Renovables (INRENARE). 1994. Resolución n ${ }^{\circ} 11$ del 29 de junio de 1994, por el cual se establece el plan de manejo del Parque Nacional Portobelo, ubicado en el distrito de Portobelo, Provincia de Colón.2 pp.

Jones, K.R., O. Venter, R.A. Fuller, J.R. Allan, S.L. Maxwell, P.J. Negret, J.E.M. Watson. 2018. One Third of Global Protected Lands is under Intense Human Pressure. Science, 360: 788-791.

Lugo, A. E., G. Cintrón. 1975. The mangrove forests of Puerto Rico and their management.: Walsh, G., Snedaker, S., Teas, H. (.), Proceedings of International Symposium on Biology and Management of Mangroves, Institute of Food and Agricultural Sciences, University of Florida, Gainesville, FL, pp. 825-846.

Lugo, A. E., E. Medina, K. McGinley. 2014. Issues and Challenges of Mangrove Conservation in the Anthropocene. Madera y Bosques 20(3):11-38.

Lugo, A. E., S. C. Snedaker. 1974. The ecology of mangroves. Annual Review of Ecological Systems 5: 39-64.

Maass, J.M., E.J. Jardel, A. Martínez-Yrízar, L.E. Calderón-Aguilera, J. Herrera, A. Castillo, J. Euán-Ávila, Equihua, M. 2010. Protected natural areas and long term ecological research in Mexico. Ecosistemas 19(2):69-83

Medrano, B., J. Hernández. 2017. Estado Actual de las Áreas Protegidas y Pautas para la Gestión de la Biodiversidad en el Salvador. Análisis, 3: $1-36$.

Ministerio de Economía y Finanzas de la República de Panamá (MEF) (2013). Decreto ejecutivo 1366 del 28 de diciembre de 2012, que modifica el decreto 43 del 16 de junio de 1999, por el cual se 
reglamentan los capítulos II y III de la ley 91 del 22 de diciembre de 1976 y se establece el ordenamiento territorial del Parque Nacional Portobelo y el Conjunto Monumental Histórico de Portobelo.

Ministerio de Ambiente de la República de Panamá (MIAMBIENTE) (2017). Resolución n ${ }^{\circ}$ DAPVS-0002-2017 del 28 de marzo de 2017, por el cual se aprueba el plan de uso público del Parque Nacional Portobelo.

Pabón-Zamora, L., J. Bezaury, F. León, L. Gill, S. Stolton, A. Groves, S. Mitchell, N. Dudley. 2008. "Valorando la Naturaleza: Beneficios de las áreas protegidas". Serie Guía Rápida, editor, J. Ervin. Arlington, VA: The Nature Conservancy. 34 pp.

Riemann, H., R. V. Santos- Álvarez, A. Pombo. 2011. El Papel de las Áreas Protegidas en el Desarrollo Local: El caso de la Península de Baja California. Gestión y Política Publica 20(1): 141-172

Sousa, W. P., P.G. Kennedy, B.J. Mitchell, B. M. Ordonez L. 2007. Supply-side ecology in mangroves: do propagule dispersal and seedling establishment explain forest structure? Ecological Monographs, 77(1), 53-76

Sousa, W.P., P.G. Kennedy, y B.J. Mitchell. 2003. Propagule size and predispersal damage by insects affect establishment and early growth of mangrove seedlings. Oecologia 135:564-575.

Weaber, P.L., G.P. Bauer, B. Jiménez. 2004. Parque Nacional San Lorenzo, El tesoro del Caribe Panameño. Editora Novo, Colombia.

Recibido 24 de marzo de 2020, aceptado 04 de abril de 2020. 